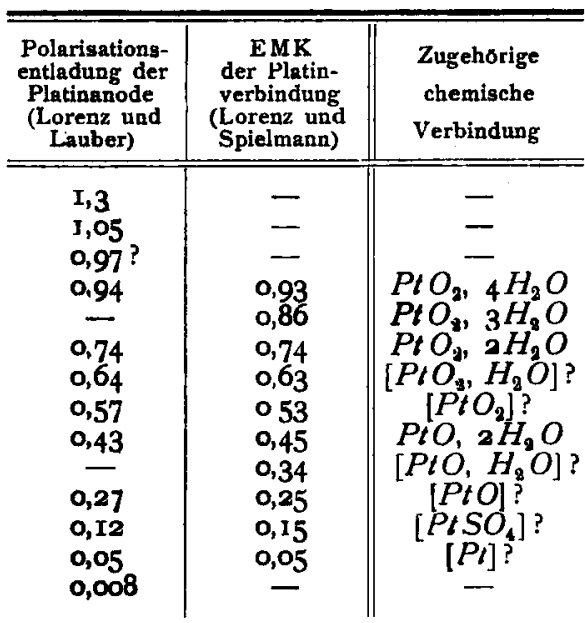

punkte zu fehlen scheinen. Diese können aber leicht abersehen sein. Immerhin ist das Resultat fur einen ersten Versuch in dieser Richtung ein aberraschendes, denn wie aus dem Vorstehenden ersichtlich ist, lassen sich alle an der Platinsauerstoffelektrode abspielenden Vorgănge bis zur Hobe von 0,94 Volt ungezwungen mittels der Oxydtheorie der Sauerstoffelektrode erklåren.
Wie sich die Erklărung der oberhalb 0,94 Volt liegenden Vorgănge gestalten wird, muß erst die Zukunft lehren. Gerade diese Erklärung wird aber erst die Aufklarung der Vorgănge in der Knallgaskette bringen. Sehr viel Beobachtungen (es sei besonders auf diejenigen von Bose, Wilsmore, Haber, Foerster hingewiesen) sprechen, besonders im Licht der hier mitgeteilten Beobachtungen dafur, daß auch die oberhalb 0,94 Volt gelegenen Punkte sich als Platinoxyde (Hydroxyde) identifizieren lassen werden, doch war es bislang nicht möglich, das betreffende Platinoxyd zu fassen, und so sind die Akten darüber immer noch nicht geschlossen. In der folgenden Mitteilung soll noch einiges Diesbezügliche erwähnt werden. Hier sei nur noch bemerkt, daß dies hier erhaltene allgemeine Resultat die von Lorenz und Hauser bereits ausgesprochene Ansicht bestätigt, nach der auch die Platinsauerstoffelektrode als eine Oxydelektrode aufzufassen ist.

Zurich, Sommersemester 1907 bis Sommersemester I 908 .

(Eingegangen: 29. April.)

\title{
METRONOMUNTERBRECHER ZUM ELEKTROMAGNETISCHEN RÜHRER FÜR GEFRIERAPPARATE.
}

\section{Von Ernst Beckmann.}

(Mitteilung aus dem Laboratorium für angewandte Chemie der Universität Leipzig.)

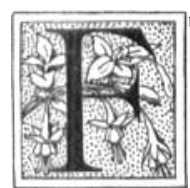

ür kryoskopische Versuche habe ich, neben einem Handrührer ${ }^{1}$ ), eine elektromagnetische Ruhrvorrichtung ${ }^{2}$ ) besonders für die Falle empfohlen, wo das Lösungsmittel bezw. die Losung wahrend des Versuches von der Außenluft vollig abgeschlossen werden soll und eine groblere Sicherheit der Resultate durch gleichmåbiges mechanisches Rahren wünschenswert erscheint. Die Versuchsanordnung ist in der Fig. 225 wiedergegeben, welche obne weiteres verståndlich sein dürfte. Der Kontakt wird durch einen Platinstift bezw. ein starkes Plättchen aus Silber bewirkt, dessen Oxyd die Elektrizităt ebenfalls leitet.

Durch ein großBeres, eine ganze Seite fallendes Inserat auf dem Umschlag der Z. f. Elektroch. ${ }^{3}$ ) wird von der Firma Ludwig $H$. Zeller, Leipzig, gegen mein Metronom mit Platinkontakt der Vorwurf erhoben, daB die Kontaktstelle allmählich durchbrenne und leicht

I) Zeitschr. f. physik. Chemie 2, 639 (I888); 7, 324 (I89I); 22, 617 (1897).

a) Zeitschr. f. physik. Chemie 21, 240 (I8g6); 44, 173 (1903).

3) Z. f. Elektroch., Bd. 15, Heft 7 und 8, vom I. und r. $_{5}$ April Igog. in Unordnung gerate, was um so fataler sei, wenn das Ruhren dann aussetze, wo die Eisausscheidung beginne.

Auf Anregung von Professor Dr. Rimbach hat die genannte Firma an Stelle des Metronomunterbrechers eine Vorrichtung mit Druckknopf gefertigt, welcher, mit einern Finger der linken Hand in richtigem Tempo niedergedruckt, einen zuverlässigen Kontakt geben soll. Durch solche Vorrichtung warde aber mein elektromagnetischer Rührer, auBer der Bequemlichkeit, eines Hauptvorzuges beraubt, nämlich der Sicherung der Resultate durch gleichmäßiges automatisches Rühren.

Ich möchte deshalb die Beibehaltung meines Metronomunterbrechers anraten, zugleich aber die Ausfährungsformen mitteilen, welche sich in den letzten Jahren als durchaus zuverlässig bewahrt haben.

Die statt der anfänglich benutzten Quecksilberkontakte eingeführten Platinunterbrecher zeigten nur so lange gelegentliches Versagen, als der Kontakt immer an derselben, feststehenden Stelle erfolgte. Bei den jetzt verwendeten Formen ist dafür gesorgt, daß die Kontaktstelle jeden Augenblick durch Verschieben bequem gewechselt werden kann. 


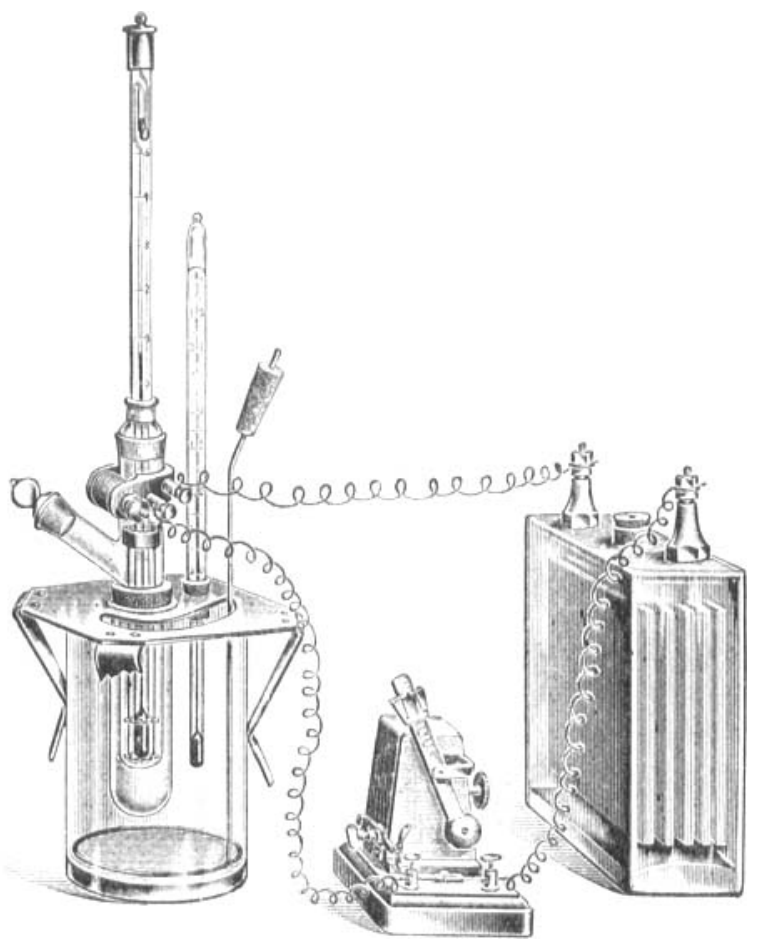

Fig. 225.

Gefrierapparat mit elektromagnetischem Rührer und Metronomunterbrecher. 1/\% wirkl. Größe.

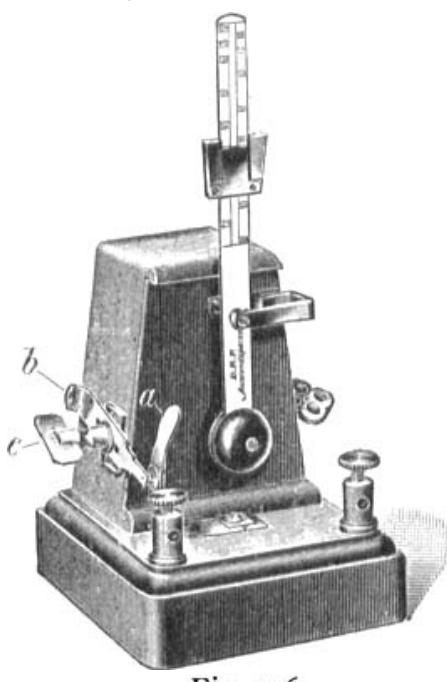

Fig. 226.

Metronomunterbrecher, 40 Minuten Gangdauer. 1/3 wirkl. Größe.

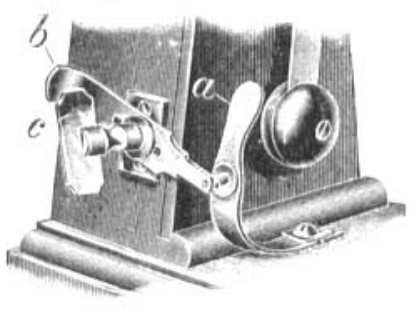

Fig. 227.

Kontaktstelle aus Fig. 226. 1/a wirkl. Größe.

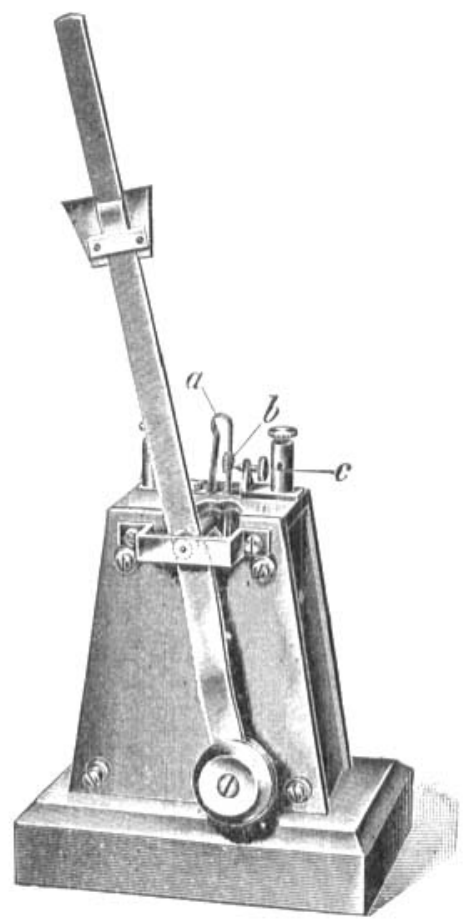

Fig. 228.

Metronomunterbrecher, 2 Stunden Gangdauer. 1/3 wirkl. Größe.
Da auch das Ablaufen des Uhrwerkes während des Versuches leicht stört, sind die fräheren Metronomen mit 20 Minuten Gangdauer durch solche ersetzt worden, welche 40 Minuten bezw. 2 Stunden Gangdauer haben.

Fig. 226 . gibt das kleinere billigere Metronom mit 4o Minuten Gangdauer wieder. In Fig. 227 ist die Kontaktstelle vergrößert abgebildet. Die Perpendikellinse des Metronoms schlägt gegen eine Feder $a$ und stellt so den Kontakt mit der Feder $b$ her, wodurch der Gang des Metronoms nicht gestort wird. Man erkennt, wie der Kontaktstift nach Lơsung der Schraube $c$ nach allen Seiten bequem verschoben werden kann.

Empfehlenswerter ist noch der in Fig. 228 wiedergegebene Unterbrecher von 2 Stunden Gangdauer. Fig. 229 läBt die innere Einrichtung erkennen. Die Kontaktstelle ist in der Fig. $23^{\circ}$ vergrößert wiedergegeben. In den Fig. 228 u. 229 ist ersichtlich, daß ein Stift der Perpendikelachse (bei $b$ in Fig. 229) das Hinund Herbewegen der Feder $a, b$ bewirkt. Das auf der Feder $a, b$ angebrachte Silberplatttchen kann durch Drehung eines die Feder durchsetzenden Schräubchens auch wahrend des Ganges bequem gegen den Platinkontaktstift $c$ verschoben werden.

Bei beiden Vorrichtungen geht der Strom, abweichend von dem anfangs verwendeten Metronomunterbrecher mit Quecksilberkontakt, nicht durch das Uhrwerk und kann deshalb dessen Gang nicht beeinträchtigen. Während

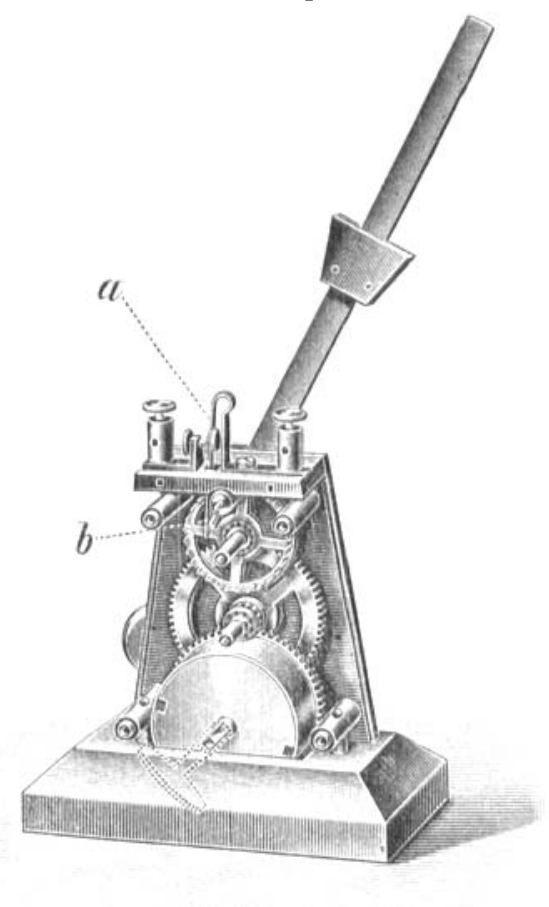

Fig. 229.

Innenansicht zu Fig. 228. $1 / 3$ wirkl. Größe. 
fraher die Kontaktfedern oben auf dem Metronomgehăuse horizontal angebracht wurden, ist jetzt eine senkrechte Stellung der Federn gewählt

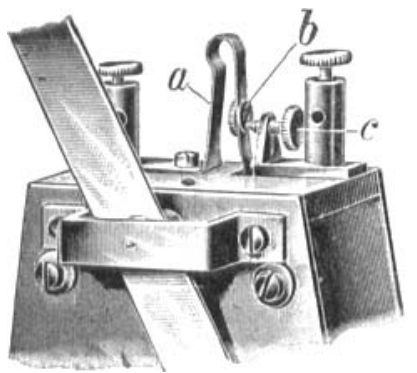

Fig. 230

Kontaktstelle aus Fig. 228. 1/2 wirk1. Größe.

worden, weil dadurch ein Erlahmen und $\mathrm{Zu}$ sammenkleben sicherer vermieden wird.

Ein Versagen dieses Unterbrechers ist auch ohne Anschalten eines elektrolytischen, zur Ab- schwächung des Oeffnungsfunkens dienenden Kondensators nicht $z u$ befürchten, hätte aber in dem Moment der Eisabscheidung besonders wenig zu bedeuten. In diesem Augenblick wird der Experimentierende zur Stelle sein, was sonst bei diesem Unterbrecher nicht notwendig ist, und kann durch Verschieben der Kontaktstelle sofort den Fehler beseitigen.

Wer sich an das Arbeiten mit dem Metronom. unterbrecher gewöhnt hat, wird die Nachteile des empfohlenen Druckknopfes nicht gering einschätzen. Jedenfalls ist letzterer, gleich dem Handrührer, weniger bequem und dabei unzuverläsșiger in bezug auf gleichmäßiges Rühren.

Die beschriebenen Metronomunterbrecher werden vom Mechaniker G. Hildebrandt des Laboratoriums für angewandte Chemie, Leipzig, Bräderstraße 34, sowie von der Firma Otto Preßler, Leipzig, geliefert.

(Eingejgangen: 4. Mai.)

\section{WISSENSCHAFTLICHE ÜBERSICHTEN.}

BERICHT ÜBER DIE ARBEITEN ÜBER KOLLOIDE.

\section{Oktober bis $3^{\text {I. Dezember } 1907 .}$} Von A. Lottermoser.

V. Auger, Hydrosol des Arsens (Compt. rend. 145, 7 18-720). Wenn eine Lösung von Arsenchlorid in absolutem Alkohol langsam in eine auf $-15^{\circ}$ abgekuhlte alkoholische Lösung von unterphosphoriger Säure gebracht wird, so entsteht unter mäBiger Temperatursteigerung, z.:B. bis auf o ${ }^{0}$, ein brauner Niederschlag, der mit Alkohol gewaschen und im Vakuum getrocknet, selbst nach längerem Äufbewahren im Vakuum (höchstens zwei Monate) in verdünnter Alkalilauge mit brauner Farbe zu einem, in der Durchsicht klaren, in der Aufsicht getrübt erscheinenden Hydrosole löslich ist. Das Hydrosol gibt mit starkem Alkali und Alkohol Niederschläge, die in Wasser löslich sind. Sauren erzeugen dagegen das irreversible Gel. Es ist nicht moglich, das Alkali vollkommen zu entfernen, ebenso kann man das Hydrosol nicht ganz von einem Gehalte an Phosphor, welcher beim Erhitzen des festen Produktes als Phosphorwasserstoff abgegeben wird, befreien. Das Hydrosol enthălt Arsen im elementaren Zustande, welches sich bei Luftzutritt in der alkalischen Flüssigkeit langsam $\mathrm{zu}$ arsenigsaurem Salze oxydiert.

Szilard ${ }^{\prime}$ ist es gelungen, einige Oxyde in Hydrosole ubberzufahren. Wenn aus verdannten Thorium- resp. Zirkoniumnitratlösungen durch verdunntes Ammoniak gefälltes Thorium- resp. Zirkoniumoxyd mit Wasser fortgesetzt aus-

I) Journ. de chim. phys. 5, 488 bis 494 . gewaschen und dekantiert wird, so setzen sich schlieflich die Oxyde nicht mehr ab, sondern bilden eine milchartige Flusssigkeit, in der Ammoniak auch nicht einmal in Spuren nachzuweisen ist. Szilard bezeichnet die Hydrosole als elektrolytfrei, da auf roo g des Ausgangsmateriales ungefahr $0,5 \mathrm{hl}$ Waschwasser verbraucht wurden. Indessen, wenn man bedenkt, wie hartnäckig Elektrolyte gerade von Oxyden festgehalten werden, erscheint dem Referenten gerade dieser Nachweis noch nicht einwandfrei vom Verfasser erbracht worden zu sein. Die erhaltenen Hydrosole sind, gegen die Kohlensäure der Luft geschützt, haltbar, alle Elektrolyte fällen das Gel aus, selbst die Kohlensäure der Luft. Einige Hydrosole hat Szilard dann auf einem Wege, den vor ihm schon Arthur Mulle ${ }^{1}$ ) benutzt hat, erhalten, indem er die reinen Oxyde in wasserhaltiger Form (oft auch die auf die oben beschriebene Weise gewonnenen Oxydhydrosole) in die Lossung ihrer Nitrate oder Chloride brachte, solange noch davon aufgenonmen wurde. Er verwandelte so $\mathrm{Th}_{2}$ durch Erhitzen mit $T h\left(\mathrm{NO}_{3}\right)_{4}$ - und $\mathrm{ThCl}_{4}$-Lơsung, $\mathrm{ZrO}_{2}$ durch $\operatorname{Zr}\left(\mathrm{NO}_{3}\right)_{4}$-Lösung und $\mathrm{UrO}_{3}$ durch $\mathrm{UrO}_{2}\left(\mathrm{NO}_{3}\right)_{2}$-Lósung in reversible Hydrosole, die vollkommen klar auch im auffallenden Lichte und auch gegen kleine Elektrolytmengen beständig sind. Auch Hițze und Licht haben

1) Ber. 39, 2857 (1906); Zeitschr. f. anorg. Chemie 52, $3^{16}$ (I907); ref. Z. f. Elektroch. 14, 63.5. 